JOURNAL OF POROUS MEDIA (BEGELL HOUSE, USA)

(EDITOR-IN-CHIEF: PROFESSOR KAMBIZ VAFAI, UNIV. OF CALIFORNIA-RIVERSIDE, USA)

https://www.begellhouse.com/journals/porous-media.html

ISSN Print: 1091-028X; ISSN Online: 1934-0508

Accepted October $22^{\text {nd }} 2020$

\title{
NUMERICAL SIMULATION AND ENERGY FLUX VECTOR VISUALIZATION OF RADIATIVE-CONVECTION HEAT TRANSFER IN A POROUS TRIANGULAR ENCLOSURE
}

K. Venkatadri ${ }^{1}$, O. Anwar Bég ${ }^{2}$, P. Rajarajeswari ${ }^{*}$, V. Ramachandra Prasad ${ }^{4}$, A. Subbarao ${ }^{5}$ and B. Md. Hidayathulla Khan ${ }^{6}$

${ }^{I}$ Department of Mathematics, Sreenivasa Institute of Technology and Management Studies, Chittoor-517127, India.

${ }^{2}$ Department of Aeronautical/Mechanical Engineering, University of Salford, School of Science, Engineering, Environment, M5 4WT, UK. ${ }^{3}$ Department of Computer Science and Engineering, Kingston Engineering College, Vellore-632014, India ${ }^{4}$ Department of Mathematics, School of Advanced Sciences, Vellore Institute of Technology, Vellore - 6322014, India. ${ }^{5}$ Department of Mathematics, Madanapalle Institute of Technology and Science, Madanapalle - 517 325, India. ${ }^{6}$ Department of Mathematics, Sir Vishveshwaraiah Institute of Science and Technology, Madanapalle - 517 325, India. *Corresponding Author: rajacse77@gmail.com

\section{ABSTRACT}

A detailed theoretical examination laminar natural convection heat flow in a triangular porous cavity with significant radiative heat transfer and porosity variation is presented. Twodimensional laminar incompressible flow is considered with the left slant and right walls are low and high temperature respectively, and the remaining (top) wall prescribed as adiabatic. The Darcy-Brinkman isotropic model is utilized, and the coupled governing equations are solved by a numerical method utilizing finite differences. Visualization of isotherms and streamlines is achieved with the method of Energy Flux Vectors (EFVs). The impacts of the different model parameters (Rayleigh number $R a$, Darcy number- $D a$, porosity- $\varepsilon$ and radiation parameter- $R d$ ) on the thermo fluid characteristics are studied in detail. The computations show that convective heat transfer is enhanced with greater Darcy parameter (permeability) which also leads to intensification in the density of energy flux vector patterns. The flow is accelerated with increasing buoyancy effect (Rayleigh number) and temperatures are also increased with greater radiative flux. Average Nusselt number is decreased with higher porosity. The simulations are relevant to hybrid porous media solar collectors.

KEYWORDS: Energy flux vectors, Darcy-Brinkman model, Thermal Radiation, Triangular Cavity, Darcy number; Nusselt number, Solar collectors.

\section{NOMENCLATURE:}

\begin{tabular}{|l|l|}
\hline$C_{p}$ & Specific heat \\
\hline$D a$ & Darcy number \\
\hline$g$ & Gravity $\left(\mathrm{m} / \mathrm{s}^{2}\right)$ \\
\hline$K$ & Permeability $\left(\mathrm{m}^{2}\right)$ \\
\hline$L$ & Length of the enclosure $(\mathrm{m})$ \\
\hline$N u$ & Nusselt number \\
\hline$p$ & Dimensional pressure $(\mathrm{Pa})$ \\
\hline
\end{tabular}




\begin{tabular}{|l|l|}
\hline$P$ & Non-dimensional pressure \\
\hline$R a$ & Rayleigh number \\
\hline$R d$ & Radiation parameter \\
\hline$t$ & Dimensional time $(s)$ \\
\hline$T$ & Temperature $(K)$ \\
\hline$T_{h}$ & Temperature of hot wall $(\mathrm{K})$ \\
\hline$T_{c}$ & Temperature of cold wall $(\mathrm{K})$ \\
\hline$(u, v)$ & Dimensional velocity components $(\mathrm{m} / \mathrm{s})$ \\
\hline$(U, V)$ & Non-dimensional velocity components \\
\hline$(x, y)$ & Dimensional coordinates $(\mathrm{m})$ \\
\hline$(X, Y)$ & Non-dimensional coordinates \\
\hline $\mathbf{G r e e k ~ S y m b o l s}$ & \\
\hline$\alpha$ & Thermal diffusivity $\left(\mathrm{m} / \mathrm{s}^{2}\right)$ \\
\hline$\beta$ & Mean absorption coefficient \\
\hline$\beta *$ & Coefficient of volume expansion $(1 / \mathrm{K})$ \\
\hline$\tau$ & Non-dimensional time \\
\hline$\rho$ & Fluid density $\left(\mathrm{kg} / \mathrm{m}^{3}\right)$ \\
\hline$\mu$ & Dynamic viscosity $(\mathrm{kgm} / \mathrm{s})$ \\
\hline$\tilde{\mu}$ & Effective dynamic viscosity $(\mathrm{kgm} / \mathrm{s})$ \\
\hline$v$ & Kinematic viscosity $\left(\mathrm{m}^{2} / \mathrm{s}\right)$ \\
\hline$\theta$ & Non-dimensional temperature \\
\hline$\kappa$ & Thermal conductivity $(\mathrm{W} / \mathrm{mK})$ \\
\hline$\lambda$ & Ratio of diffusivities \\
\hline$\varepsilon$ & Porosity \\
\hline$\sigma=5.67037$ & Stefan-Boltzmann constant \\
$\mathbf{x} 10^{-8} \mathrm{~W} \mathrm{~m}^{-2} \mathrm{~K}^{-4}$ & \\
\hline$\rho C$ & Heat capacitance $(\mathrm{J} / \mathrm{K})$ \\
\hline$\psi$ & Dimensionless stream function \\
\hline$W$ & Dimensionless vorticity \\
\hline Subscripts & Hot \\
\hline$h$ & Cold \\
\hline$c$ & \\
\hline
\end{tabular}

\section{INTRODUCTION}

Recently significant research activity has been conducted in natural convection in triangular cavities owing to applications in for example solar collector systems, ventilation of buildings with inclined roofs, semi-conductor melts etc. Flow within an enclosure is an intriguing 
branch of fluid dynamics and features many sophisticated phenomena including vortices, boundary layers, pitchfork bifurcation, cells etc. In natural convection the dominant driving force is thermal buoyancy. In the case flows driven by a lid (or wall), the motion of one or more walls that define the cavity can also mobilize convection patterns. A substantial body of literature is available on natural convection flows in different geometrically shaped enclosures with straight boundaries (e.g. trapezoidal, triangular, rectangular and square [1, 2]). In many industrial applications the heat transfer in non-square enclosures (triangular) heating of one side in particular, is of great interest [3]-[4]. In these and other studies, owing to the nonlinearity of the governing conservation equations, numerical methods must be employed to generate solutions for the velocity and temperature fields. In recent years engineers have deployed Lattice Boltzmann methods [5], finite volume SIMPLE algorithms [6], variational finite element methods [7],incompressible smoothed particle hydrodynamics (ISPH) methods [8] and numerous other techniques including finite difference methods (FDM). The last of these methods is generally less expensive computationally and algebraically simpler to implement. Venkatadri et al. [9] used the finite difference (technique) method (FDM) to simulate numerically the influence of phase change (melting)heat transfer on magnetohydrodynamic natural convection of bottom wall heating of enclosure, noting that a greater heat transfer rate is achieved for uniform heating of the base wall compared with nonuniform heating. Bhardwaj et al. [10] also used a FDM to compute the entropy generation in a right-angled triangular wavy enclosure and noted that the undulation of the wall enclosure elevates the Nusselt number $(\mathrm{Nu})$ by 53\%, at Darcy number of 0.01 and Rayleigh number of 1 million as compared to the straight wall case. Akinsete and Coleman [11] used an alternating direction implicit code to analyse the 2D laminar buoyancy convection in a long, horizontal, right-angled geometry, triangular cavity containing air. Triveni et al. [12] adopted ANSYS FLUENT finite volume code to model the heat transfer through buoyancy driven flow in a triangular (right-angle at left wall) cavity filled with waterwith partially heating and cooling. Rahman et al. [13] employed a finite element (FE) method to investigate double diffusive convective flow in a wavy-walled (corrugated) triangular enclosure, observing that multiple circulations are generated at the lowest Prandtl number and that with greater wavelength and Rayleigh number both heat and mass transfer at the walls are enhanced. Kaluri et al. [14] simulated computationally the thermal convection flow for various aspect rations (AR) of an enclosure (rectangular) in the presence of different thermal boundary conditions and noted that elevation in uniform heating produces an exponential decrease 
in Nusselt numbers. Other numerical studies of triangular enclosure free convection flows are provided in [15]-[16].

In many industrial and manufacturing processes, porous media arise. They provide a relatively simple, inexpensive but effective method for regulating flows and transport phenomena. The classical approach in the simulation of transport in permeable media is the Darcy model which assumes a linear pressure drop across the material. This provides acceptable accuracy for lowvelocity percolation wherein viscous hydrodynamic forces dominate over inertial forces. Mathematical models of Newtonian convection in Darcian permeable media have stimulated extensive attention largely motivated by insulation, architectural and solar energy applications. Representative studies include [17]-[20] all of which have shown that altering permeability (modification in Darcy number) markedly modifies the velocity and temperature distributions. These studies assume local thermal equilibrium (LTE) exists between the fluid and permeable medium i.e. the uniform temperatures on solid and fluid phases. They however neglect porosity effects. Whereas permeability relates to the hydraulic conductivity of a porous medium, porosity (or void fraction) is defined as the ratio of the volume of all the pores in a material to the volume of the whole material. Several investigations have considered porosity variation in free convection flows. These include Vafai [21], Hong et al. [22] and Prasad et al. [23].

In the present study, a mathematical model is presented for laminar steady-state natural convective heat flow in a permeable triangular enclosure. A Darcy-Brinkman model is adopted in which porosity variation is also considered. Thermal radiation heat transfer is also included owing to its significance in solar collector systems, for which Rosseland's diffusion flux model is employed [24, 25].The enclosure has inclined sidewalls which are kept at constant temperature, and the remaining (top) wall is prescribed as adiabatic. Using a vorticity stream function formulation, the conservation equations for mass, momentum and energy are rendered nondimensional and solved computationally with a marker and cell (MAC) finite difference method with incremental steps. Hooman's [26] energy flux vectors (EVF) method is implemented to visualize thermal characteristics in the triangular porous cavity. This technique is superior to the earlier heat line visualization approach of Bejan [27] since it does not require algebraic equations to be solved and is also applicable to more complex geometries. To the best of our knowledge, the buoyancy driven flow in a triangular porous enclosure (with porosity variation, following Vafai 
[21]) including significant thermal radiation and with Hooman's heat flow visualization EFV method has not been studied thus far in the scientific literature. In the subsequent sections, the mathematical model of the considered problem is discussed in detail, with full numerical solutions, validation and graphical solutions given.

\section{MATHEMATICAL MODEL}

The physical regime under investigation comprises two-dimensional right-angled triangular cavity geometry, enclosed by three thermal walls. The top wall is thermally insulated, and the inclined and vertical walls are isothermal and prescribed different temperatures. The geometry analyzed is depicted in Fig. 1 and contains an incompressible porous medium. The enclosure slant wall and right walls are sustained at temperatures, $T_{c}$ and $T_{h}\left(\right.$ i.e. $\left.T_{c}<T_{h}\right)$ respectively. No-slip conditions are enforced on all the walls (boundaries).

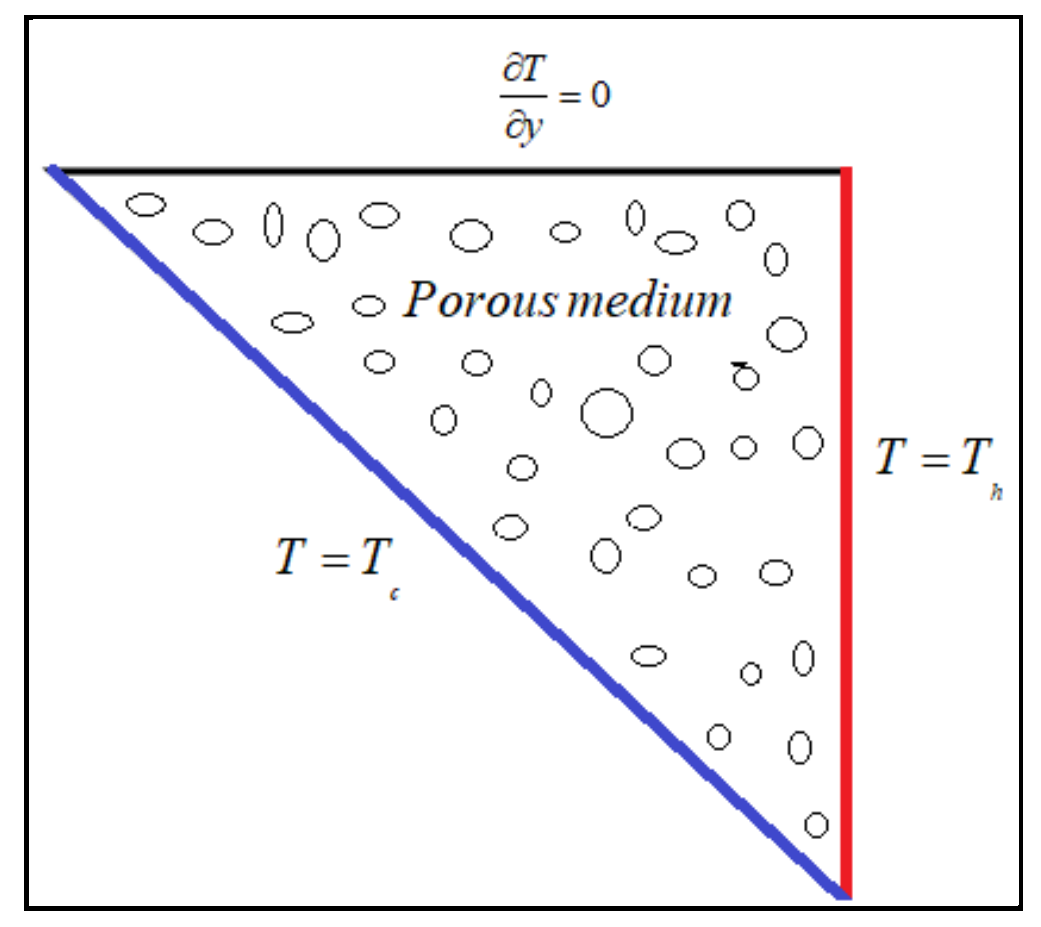

Fig. 1. Schematic representation of problem

The mathematical model is developed for the natural convection in a permeable triangular enclosure based on the following assumptions. 
- Fluid is assumed to be incompressible and Newtonian.

- The no-slip boundary condition is assumed at the solid boundaries

- The fluid flow is assumed to be laminar and two dimensional.

- The thermo-physical properties of the fluid (except the density variation in the buoyancy term) are all considered to be constant. The linear Boussinesq approximation is invoked to relate the variation of density with temperature in the body force term.

- The temperature of the fluid phase is equal to the temperature of the solid phase and solid matrix fibers and fluid in the porous medium are in local thermal equilibrium (LTE).

- Radiation heat transfer is considered for optically thick fluid.

Within the framework of the afore-mentioned assumptions, the governing equations for the present study (incorporating the Brinkman modified-Darcy flow model) may be expressed in dimensional form as follows

$\frac{\partial u}{\partial x}+\frac{\partial v}{\partial y}=0$

$-\frac{\partial p}{\partial x}-\frac{\mu}{K} u+\tilde{\mu}\left(\frac{\partial^{2} u}{\partial x^{2}}+\frac{\partial^{2} u}{\partial y^{2}}\right)=0$

$-\frac{\partial p}{\partial y}-\frac{\mu}{K} v+\tilde{\mu}\left(\frac{\partial^{2} v}{\partial x^{2}}+\frac{\partial^{2} v}{\partial y^{2}}\right)+\rho \beta g\left(T-T_{0}\right)=0$

$\frac{\partial T}{\partial t}+\frac{1}{\varepsilon}\left\{u \frac{\partial T}{\partial x}+v \frac{\partial T}{\partial y}\right\}=\frac{k}{\rho C_{p}}\left\{\frac{\partial^{2} T}{\partial x^{2}}+\frac{\partial^{2} T}{\partial y^{2}}\right\}-\frac{1}{\rho C_{p}}\left(\frac{\partial q_{r x}}{\partial x}+\frac{\partial q_{r y}}{\partial y}\right)$

The effective dynamic viscosity $\tilde{\mu}$ of the fluid in the porous channel is defined by $\tilde{\mu}=\frac{\mu}{\varepsilon}$ under the Darcy-Brinkman model. Rosseland's approximation is used for thermal radiation [25]:

$q_{r x}=-\frac{4 \sigma}{3 \beta} \frac{\partial T^{4}}{\partial x}, q_{r y}=-\frac{4 \sigma}{3 \beta} \frac{\partial T^{4}}{\partial y}$

Here $\sigma$ is the Stefan-Boltzmann constant $\left(=5.67037 \times 10^{-8} \mathrm{~W} \mathrm{~m}^{-2} \mathrm{~K}^{-4}\right)$ and $\beta$ is the mean absorption coefficient. The following transformation variables are invoked to make equations (1) - (4) dimensionless: 


$$
\begin{aligned}
& \tau=\frac{t \alpha}{L^{2}}, U=\frac{u L}{\alpha}, \quad V=\frac{v L}{\alpha}, \quad X=\frac{x}{L}, Y=\frac{y}{L} \\
& P=\frac{p K}{\mu \alpha}, \theta=\frac{T-T_{0}}{T_{h}-T_{c}}, \theta=\frac{T_{s}-T_{0}}{T_{h}-T_{c}} \\
& R a=\frac{g \beta\left(T-T_{c}\right) L^{3} P r}{v^{2}}, D a=\mathrm{K} / \mathrm{L}^{2}
\end{aligned}
$$

By using (6), equations (1) - (3), become:

$$
\begin{aligned}
& \frac{\partial U}{\partial X}+\frac{\partial V}{\partial Y}=0 \\
& U=-\frac{\partial P}{\partial X}+\frac{D a}{\varepsilon}\left(\frac{\partial^{2} U}{\partial X^{2}}+\frac{\partial^{2} U}{\partial Y^{2}}\right) \\
& V=-\frac{\partial P}{\partial Y}+\frac{D a}{\varepsilon}\left(\frac{\partial^{2} V}{\partial X^{2}}+\frac{\partial^{2} V}{\partial Y^{2}}\right)+\text { Da.Ra } \theta
\end{aligned}
$$

Stream function $\psi$ and vorticity $W$ are defined in dimensional form as:

$$
\begin{aligned}
& V=-\frac{\partial \psi}{\partial X}, U=\frac{\partial \psi}{\partial Y} \text { and } w=\frac{\partial V}{\partial X}-\frac{\partial U}{\partial Y} \\
& w=-\nabla^{2} \psi
\end{aligned}
$$

The vorticity and stream functions defined in Eqns. (10-11) are implemented in Eqns. (7-9) and Eqn. (4) and the resulting non-dimensional momentum and energy conservation equations emerge as follows:

$$
\begin{aligned}
& w=\frac{D a}{\varepsilon}\left(\frac{\partial^{2} w}{\partial X^{2}}+\frac{\partial^{2} w}{\partial Y^{2}}\right)+D a \cdot R a \frac{\partial \theta}{\partial X} \\
& \varepsilon \frac{\partial \theta}{\partial \tau}+U \frac{\partial \theta}{\partial X}+V \frac{\partial \theta}{\partial Y}=\varepsilon\left(1+\frac{4 R d}{3}\right)\left(\frac{\partial^{2} \theta}{\partial X^{2}}+\frac{\partial^{2} \theta}{\partial Y^{2}}\right)
\end{aligned}
$$

The boundary conditions as shown in Fig. 1 are as follows:

$$
\begin{aligned}
& \psi=U=V=0, \theta=0 \text { on the slant wall } \\
& \psi=U=V=0, \theta=1 \text { on the right vertical wall }
\end{aligned}
$$




$$
\psi=U=V=0, \frac{\partial \theta}{\partial y}=0 \text { on the other (top) wall }
$$

The estimation of heat transfer rate along the hot wall is more significant and is defined with the radiative effect incorporated as follows:

Local Nusselt number $N u=-\left(1+\frac{4 R d}{3}\right)\left(\frac{\partial \theta}{\partial X}\right)_{X=0}$ and Mean $N u_{M}=-\int_{0}^{1}\left(\frac{\partial \theta}{\partial X}\right) d Y$

The details of energy flux vectors and computational procedure are comprehensively discussed in [36]. The energy flux vectors $(\bar{E})$ can be obtained via post processing without any rigorous solution procedure. The energy flux vector $(\bar{E})$ is defined as follows:

$$
\bar{E}=\left(\frac{\partial \psi}{\partial Y} \theta-\left(1+\frac{4 R d}{3}\right) \frac{\partial \theta}{\partial X}\right) e_{X}+\left(-\frac{\partial \psi}{\partial X} \theta-\left(1+\frac{4 R d}{3}\right) \frac{\partial \theta}{\partial Y}\right) e_{Y}
$$

\section{MAC COMPUTATIONAL METHOD AND VERIFICATION}

The non-dimensional coupled partial differential equations (11) - (13) under the boundary conditions (14) are solved numerically. The stream function is computed from the Poisson equation (11) with boundary conditions (14). These equations are solved by the MAC method (with a finite difference technique). The complete procedure and numerical scheme have been described in detail previously by Venkatadri et al. $[9,36]$. This numerical scheme achieves high accuracy in simulating viscous fluid flow problems and is a robust alternative to other numerical methods such as the FEM (Finite Element Method), CVFE (Control Volume Finite Element) etc. Further details of the computational methodology are given in [28-32]. The MAC solver has been coded in an in-house MATLAB-based platform and has been validated with earlier numerical studies [33-35]. Streamlines and isotherms are compared with the numerical data of Basak et al. [37] and depicted in Fig. 2. Table.1 shows the mean Nusselt number at the hot wall for a wide range of $R a$ (i.e $R a=10^{3}-10^{6}$ ). Very good correlation with the finite element solutions in [37] is demonstrated, confirming the accuracy of the MAC code.

\section{RESULTS AND DISCUSSION}

Analysis of energy flux vectors (EFVs) of convective flow in a triangular permeable enclosure in presence of LTE is conducted. The simulations are conducted over a wide range of parameter values i.e. Rayleigh number $(R a=50-1000)$, Darcy number $(D a=0.1-0.0001)$, porosity 
parameter $\varepsilon(1.2-1.6$ i.e. $1.2 \%$ to $1.6 \%$ void percentage) and thermal radiation parameter ( $R d=1-$ 4). $R d$ values are based on the original study of Cess [38] which have also been verified more recently by Kuharat et al. [25]. The obtained computational results are presented in the form of contour plots via streamlines, energy flux vector, isotherms and local and average Nusselt number for variation in these parameters, $R a, D a, \varepsilon$ and $R d$.

Figs. 3-4 present the influence of Darcy parameter ( $D a=0.0001-0.1)$ on streamlines (left), isotherms (centre) and energy flux vectors (right) for $R a=100, R d=1, \varepsilon=1.2 \%$. Thermal buoyancy is therefore quite weak $(R a=100)$ and conductive and radiative heat transfer modes contribute equally $(\mathrm{Rd}=1)$ [38]. In fig. 3, very low permeability is considered i.e. $D a=0.0001$, $D a=0.001$. In fig. 4 much higher permeability (orders of magnitude higher than in Fig. 3) is studied i.e. $D a=0.01,0.1$. In Fig. 4 , at $D a=0.0001$, the thermal conduction mechanism dominates, and the medium permeability is extremely low (greater surface area of the solid matrix fibers is present which boosts thermal conduction). The streamlines exhibit a single enlarged vortex structure within the triangular cavity. With increasing permeability $(D a=0.001)$ this vortex is further expanded laterally towards the upper left corner of the triangular geometry. At $D a=0.0001$, temperature contours are parallel to the vertical wall. With enhancement in $D a$, permeability is elevated, and the fluid can percolate more easily in the porous medium as the Darcian drag force is reduced. The magnitudes of the temperature contours increase, and they are distorted at the upper region of the vertical wall and also significantly along the upper horizontal boundary. The convective heat transfer rate in the enclosure is accentuated with an increase in $D a$. The flow of heat in the porous triangular enclosure can be further visualized via energy flux vectors (EVFs). The EFV's commence from the hot regime (vertical wall) and are directed towards the low temperature regime (inclined wall) and clearly reveal the flow of heat patterns within the enclosure. The domination of conductive heat transfer at low Darcy number can be noticed from the energy flux vectors patterns. The enhancement of $D a$ causes the clustering of energy flux vector patterns from the hot wall towards the inclined wall and generates a passive thermal zone in which thermal energy is circulated. The flux vectors are somewhat straighter in the upper zone but are more significantly distorted in the lower zone where a cell is generated which is skewed towards the triangular boundary. There is also a minor influence on heat flow at the upper horizontal wall. Clearly a change in permeability exerts a non-trivial impact on thermofluid characteristics in the triangular enclosure. In fig. 4 (upper plots), for $D a=0.01$, yet further expansion in the central 
vortex is observed compared with the corresponding streamline plots in figs 3 for lower Darcy numbers. At $D a=0.1$ (highest permeability considered) there is continued growth in the vortex and a relaxation in the streamlines at the vertical wall (lower region) with a constriction along the triangular wall. Evidently there is a significant modification in momentum distribution in the regime achieved with different permeabilities. Increasing Darcy number to $D a=0.01$ and 0.1 , continues to induce a more prominent distortion in the isotherm profiles which become yet more nonlinear a short distance away from the vertical boundary and the upper horizontal boundary; however they remain largely parallel to the slope boundary. Heat distribution is clearly substantially morphed in the enclosure with increasing permeability. The largest Darcy number corresponds to a suppression in thermal conduction and an elevation in thermal convection mode in the regime. The energy flux vector plots also exhibit progressively greater alteration in the cell computed in fig. 3 at $D a=0.001$. At $D a=0.01$ (fig 4) the cell is expanded laterally towards the inclined boundary and is contracted in the vertical direction. This effect is further amplified at $D a$ $=0.1$ where the cell occupies the greater portion of the core zone in the enclosure. The angle of impingement of the flux vectors on the sloped boundary becomes increasingly sharper with highest Darcy number.

Fig.5 exhibits profiles of the streamlines, isotherms and energy flux vectors for different values of thermal Rayleigh number for $D a=0.0001, R d=1, \varepsilon=1.2$. At $R a=100$ thermal buoyancy effects are relatively weak and the temperature contours appear within the enclosure largely as parallel lines. As $R a$ increases (i.e. $R a=100,500$ and 1000), thermal buoyancy is enhanced significantly (relative to viscous hydrodynamic force) and induces a distortion in the distribution of the isotherm contours which are detracted from the hot wall and magnitudes are increased. Heat transfer is therefore exacerbated in the regime. The streamlines also exhibit a mono circulation zone within the triangular cavity. With the increase of $R a$, the initial dominance by thermal conduction is shifted to thermal convection dominance. With regard to the energy flux vectors, inspection of Fig. 5 shows that the energy flux vectors visualize the heat flow direction and position; the heat generally moves from the high temperature (vertical wall) to the low temperature (inclined) wall. As seen, the energy flux vectors exhibit a single rotating zone topology. The stronger circulation shows that higher heat transfer rate occurs in this region. As Rayleigh number decreases the energy flux vectors density diminishes due to the reduction in thermal buoyancy (relative to viscous hydrodynamic force). 
Fig. 6-8 Exhibits the effect of thermal Rayleigh number $R a$, Darcy parameter $D a$, thermal radiation flux $R d$ and porosity $\varepsilon$. The impact of porosity on average Nusselt number with various values of Rayleigh number for $D a=0.0001, R d=1$ is shown in Fig. 6. As a general trend, the average Nusselt number increases as $R a$ increases. Higher Rayleigh number implies stronger thermal buoyancy and hence the net fluid flow driving force increases. This boosts the transfer of thermal energy to the triangular boundaries from the fluid in the enclosure. However, the average Nusselt number decreases with increasing porosity since less solid fibers are available for thermal energy transfer via conduction with greater presence of voids. Designers must therefore judiciously select appropriate porous media for solar collector design in order not to suppress heat transfer rates to strongly while regulating the flow circulation. The influence of Darcy number on average Nusselt number is depicted in Fig.7. The convective heat transfer is gradually increased with an elevation in Darcy parameter i.e. greater permeability results in intensification in transfer of thermal energy from within the enclosure to the enclosure walls. This results in denser energy flux vectors patterns. Finally, the thermal radiation is found to also exert a marked effect on enhancement of heat transfer, at any value of Rayleigh number, as seen in Fig. 8. The stronger radiative flux energizes the fluid in the enclosure (corresponding to greater solar flux) which leads to a boost in the transfer of heat to the boundaries. For the case of $R d=1$ there is an equivalence of thermal conduction and radiative heat transfer and average Nusselt number is minimized for this scenario. Overall, the MAC simulations provide a good insight into the heat and momentum characteristics in the enclosure and their modification with radiative flux, medium porosity, permeability and thermal buoyancy.

\section{CONCLUSIONS}

The natural convective flow and radiation heat transfer in a triangular porous enclosure has been examined theoretically and numerically in this article. The enclosure base wall is prescribed a uniform high temperature and the top wall is maintained as thermally insulated. The inclined left wall is sustained at constant low temperature. The governing dimensionless conservation equations are solved in vorticity form using a MAC finite difference method [9]. Heat flow analysis is also conducted within the trapezoidal enclosure using Hooman's EFV method [26]. The computations have shown that: 
(i) Low average Nusselt numbers (wall heat transfer rates) and velocities are obtained for low Rayleigh number $(R a=50)$. However multiple circulations and multiple cores of energy flux vectors are found at higher Rayleigh number $\left(R a=10^{3}\right)$ and average Nusselt number and velocities are increasing functions of the Rayleigh number $R a$.

(ii) Significant elevation in the average Nusselt number is also computed with increasing radiation parameter $R d$.

(iii) It is also noteworthy that thermal radiative flux, Darcy number, porosity parameter and buoyancy (as simulated via $R d, D a, \varepsilon$ and $R a$ ) are very good control parameters for heat and momentum characteristics inside triangular porous cavities.

(iv) Careful selection of these parameters can achieve optimal heat transfer rates in for example solar enclosures.

The present simulations have demonstrated that the MAC finite difference code is a good computational solver for solar enclosure simulations. Future studies may consider wavy boundaries (corrugated walls), more complex radiative models (e.g. Schuster-Schwartzchild 2flux model), nanofluids and non-Newtonian working fluids.

\section{REFERENCES}

[1] Wu W., Ewing D., Ching C.Y. (2006). The effect of the top and bottom wall temperatures on the laminar natural convection in an air-filled square cavity, Int. J. Heat Mass Transfer, Vol. 49, pp. 1999-2008. https://10.1016/j.ijheatmasstransfer.2005.11.027

[2] Ramakrishn D., Basak T., Roy S., Momoniat E. (2014). Analysis of thermal efficiency via analysis of heat flow and entropy generation during natural convection within porous trapezoidal cavities, Int. J. Heat Mass Transfer, 77, pp. 98-113. https://10.1016/j.ijheatmasstransfer.2014.04.002

[3] Flack, R. D., Konopnicki, T. T., and Rooke, J. H. (1979). The measurement of natural convective heat transfer in triangular enclosures. ASME. J. Heat Transfer. November 1979; 101(4): 648-654. https://doi.org/10.1115/1.3451051

[4] Sameh E. Ahmed, A. M. Rashad and Rama Subba Reddy Gorla (2013). Natural convection in triangular enclosures filled with nanofluid saturated porous media,AIAA J Thermophysics Heat Transfer, 27 (4) 1-10. https://doi.org/10.2514/1.T4029

[5] Kefayati, G.H.R., S.F. Hosseinizadeh, M. Gorji, H. Sajjadi, 2011. Lattice Boltzmann simulation of natural convection in tall enclosures using water/ $\mathrm{SiO}_{2}$ nanofluid, Int. Comm. Heat and Mass Transfer, 38, 6, 798-805. 
[6] Kuharat, S., O. Anwar Bég,Ali Kadir, B. Vasu, Tasveer A. Bég and W.S. Jouri, 2019. Computation of gold-water nanofluid natural convection in a three-dimensional tilted prismatic solar enclosure with aspect ratio and volume fraction effects, Nanoscience and Technology- An International Journal. In press

[7] Bhargava, S. Sharma, P. Bhargava, O. Anwar Bég and A. Kadir, 2017. Finite element simulation of nonlinear convective heat and mass transfer in a micropolar fluid-filled enclosure with Rayleigh number effects, Int. J. Applied Computational Mathematics, 3 (2), 1347-1379.

[8] Zehba, A.M.A. and A. S. Raizah, 2016. Double-diffusive natural convection in an enclosure filled with nanofluid using ISPH method, Alexandria Engineering Journal, 55, 3037-3052.

[9] K. Venkatadri, S. Abdul Gaffar, M. Suryanarayana Reddy, V. Ramachandra Prasad, B. Md. Hidayathulla Khan and O. Anwar Bég (2020). Melting heat transfer analysis on magnetohydrodynamics buoyancy convection in an enclosure: a numerical study, J. Appl. Comput. Mech., 6(1) 52-62.https://10.22055/JACM.2019.28761.1504

[10] Bhardwaj S., Dalal A., Pati S. (2015). Influence of wavy wall and non-uniform heating on natural convection heat transfer and entropy generation inside porous complex enclosure, Energy, Vol. 79, pp. 467-481. https://10.1016/j.energy.2014.11.036

[11] V.A. Akinsete T.A. Coleman (1979). Heat transfer by steady laminar free convection in triangular enclosures, Numerical methods in thermal problems; Proceedings of the First International Conference, Swansea, Wales, July 2-6.

[12] Triveni M.K., Sen D., Panua R.S. (2014). Laminar natural convection for thermally active partial side walls in a right-angled triangular cavity, Arab. J. Sci. Eng., Vol. 39, pp. 90259038. https://10.1007/s13369-014-1418-7

[13] Rahman M.M., Saidur R., Mekhilef S., Uddin M.B., Ahsan A. (2013). Double-diffusive buoyancy induced flow in a triangular cavity with corrugated bottom wall: effects of geometrical parameters, Int. Comm. Heat Mass Transfer, Vol. 45, pp. 64-74. https://10.1016/j.icheatmasstransfer.2013.04.002

[14] Kaluri R.S., Anandalakshmi R., Basak T. (2010). Bejan's heat line analysis of natural convection in right-angled triangular enclosures: effects of aspect-ratio and thermal boundary conditions, Int. J. Thermal Sciences, Vol. 49, pp. 1576-1592. DOI: https://10.1016/j.ijthermalsci.2010.04.022

[15] Lei, C. \& Patterson, J. C. (2005) Unsteady natural convection in a triangular enclosure induced by surface cooling. Intl. J. Heat Fluid Flow26, 307-321.

[16] Holtzman, G.A., Hill, R.W. and Ball, K.S. (2000). Laminar natural convection in isosceles triangular enclosures heated from below and symmetrically cooled from above, ASME J. Heat Transfer, 122, 485-491.

[17] A. Bejan, D. Poulikakos (1982). Natural convection in an attic-shaped space filled with porous material, ASME J. Heat Transfer 104, 241-247.

[18] T. Basak, C. Thirumalesha, S. Roy (2008). Finite element simulations of natural convection in a right-angle triangular enclosure filled with a porous medium: effects of various thermal boundary conditions, J. Porous Media 11, 159-78.

[19] Y. Varol (2011). Natural convection in porous triangular enclosure with a centered conducting body, International Communications in Heat and Mass Transfer, 38, 368-376.

[20] R. Chowdhury et al. (2015). Natural convection in porous triangular enclosure with a circular obstacle in presence of heat generation, American Journal of Applied Mathematics, 3, 5158. 
[21] Vafai, K. (1984), Convective flow and heat transfer in variable porosity media, J. Fluid Mechanics, 147, 233-259.

[22] Hong, J. T., Yamada, Y.and Tien, C. L. (1987). Effects of non-Darcian and non-uniform porosity on vertical plate natural convection in porous media, ASME J. Heat Transfer, 109, 356-362

[23] VR Prasad, B Vasu, O. Anwar Bég, RD Parshad. (2012). Thermal radiation effects on magnetohydrodynamic free convection heat and mass transfer from a sphere in a variable porosity regime, Communications in Nonlinear Science and Numerical Simulation 17 (2), 654-671.

[24] K. Ghachem et al. (2015). Numerical study of combined natural convection and radiation in three- dimensional solar thermal collector: focus on the inclination effect on heat transfer, American Journal of Modern Energy, 1(2): 44-51.

[25] S. Kuharat, O. Anwar Bég, A. Kadir and M. Shamshuddin (2019). Computational study of heat transfer in solar collectors with different radiative flux models, Heat Transfer Asian Research, 1-30 (2019). DOI: 10.1002/htj.21418 (30 pages)

[26] Hooman, H. 2010. Energy flux vectors as a new tool for convection visualization. Int. $J$. Numer. Methods Heat Fluid Flow, 20, 240-249.

[27] Kimura, S. and Bejan, A. 1983. The "heat line" visualization of convective heat transfer. ASME J. Heat Transfer, 105, 916-91.

[28] Ertuk E, Corke TC and Gokcol C. (2005) Numerical solution of 2-D steady incompressible driven cavity flow at high Reynolds number, Int. J. Numerical Methods in Fluids, 48, 747774.

[29] Ertuk E and Gokcol O (2007). Fine grid numerical solution of triangular cavity flow, The European Physical Journal - Applied Physics, 38, 97-105.

[30] Ertuk E and Dursun B. (2007). Numerical solutions of 2-D steady incompressible flow in a driven skewed cavity, ZAMM-Journal of Applied Mathematics and Mechanics, 87, 377-392.

[31] Sheremet, M.A. and Pop, I. (2014). Natural convection in a square porous cavity with sinusoidal temperature distributions on both side walls filled with a nanofluid: Buongiorno's mathematical model, Transport in Porous Media, 105 (2) 411-429.

[32] Sheremet, M.A. and Pop, I. (2015). Natural convection in a wavy porous cavity with sinusoidal temperature distributions on both side walls filled with a nanofluid: Buongiorno's mathematical model, ASME Journal of Heat Transfer, 137, 072601.

[33] D. de Vahl Davis (1983). Natural convection of air in a square cavity: a benchmark solution, Int. J. Numer. Meth. Fluids, vol. 3, pp. 249-264.

[34] M. T. Manzari (1999). An explicit finite element algorithm for convective heat transfer problems, Int. J. Numer. Meth. Heat Fluid Flow, vol. 9, pp. 860-877.

[35] D. C. Wan, B. S. V. Patnaik and G. W. Wei (2001). A new benchmark quality solution for the buoyancy-driven cavity by discrete singular convolution, Numerical Heat Transfer, Part $B$, 40: 199-228.

[36] K. Venkatadri, O. Anwar Beg, P Rajarajeswari, V. Ramachandra Prasad (2020), Numerical simulation of thermal radiation influence on natural convection in a trapezoidal enclosure: heat flow visualization through energy flux vectors. International Journal of Mechanical Sciences 171, 105391.

[37] Tanmay Basak, S. Roy, Ch. Thirumalesha (2007). Finite element analysis of natural convection in a triangular enclosure: Effects of various thermal boundary conditions, Chemical Engineering Science, 62; 2623-2640. 
[38] Cess, R.D., (1966) The interaction of thermal radiation with free convection heat transfer. International Journal of Heat and Mass Transfer, 9, 1269-1277.

\section{TABLES \& FIGURES}

Table. 1. Comparison of average Nusselt number (Nu) with $\operatorname{Pr}=0.71$ (air)

\begin{tabular}{cccccc}
\hline & & Ref. [33] & Ref. [34] & Ref. [35] & Present study \\
$\mathrm{Ra}$ & $\mathrm{Nu}$ & & & FEM & (FDM) \\
\hline $10^{3}$ & Average & 1.12 & 1.074 & 1.117 & 1.1185 \\
$10^{4}$ & Average & 2.243 & 2.084 & 2.254 & 2.2526 \\
$10^{5}$ & Average & 4.52 & 4.3 & 4.598 & 4.5907 \\
$10^{6}$ & Average & 8.8 & 8.743 & 8.976 & 8.9905 \\
\hline
\end{tabular}
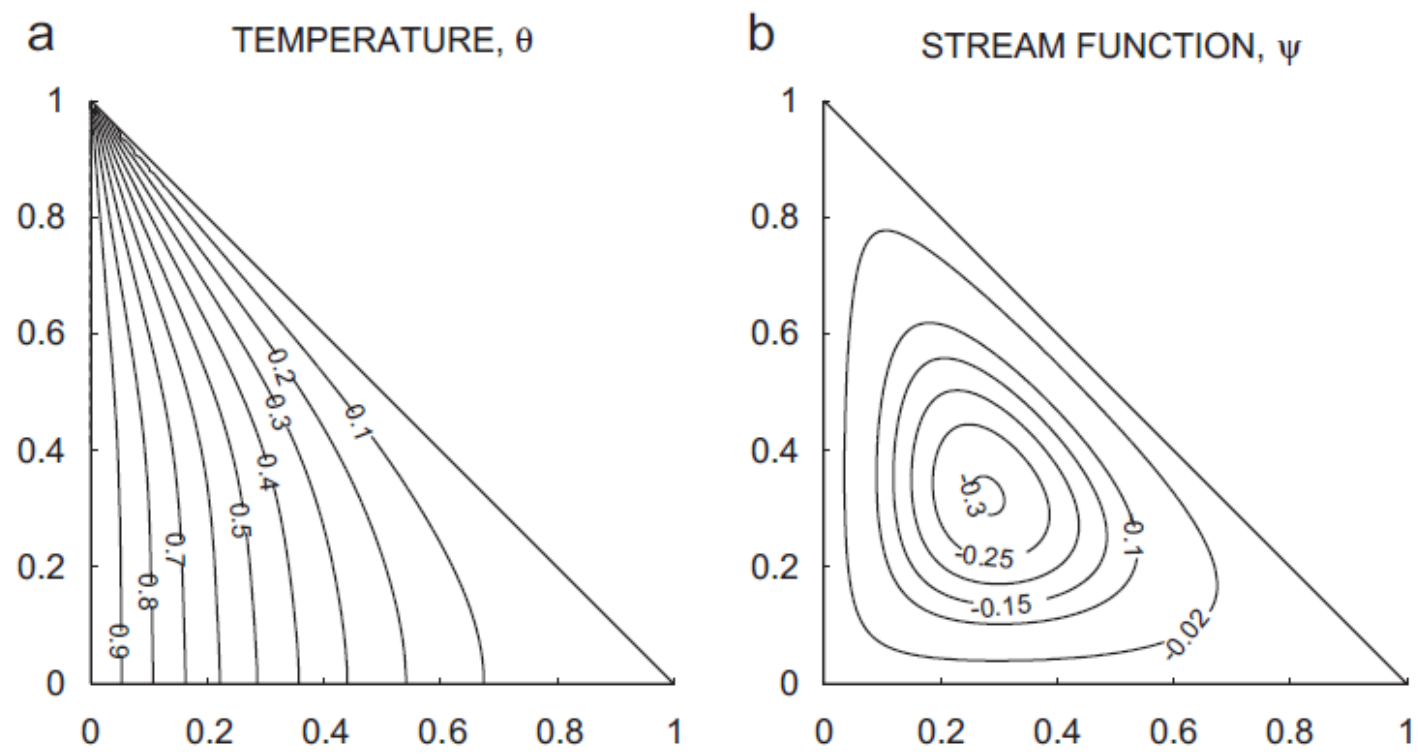


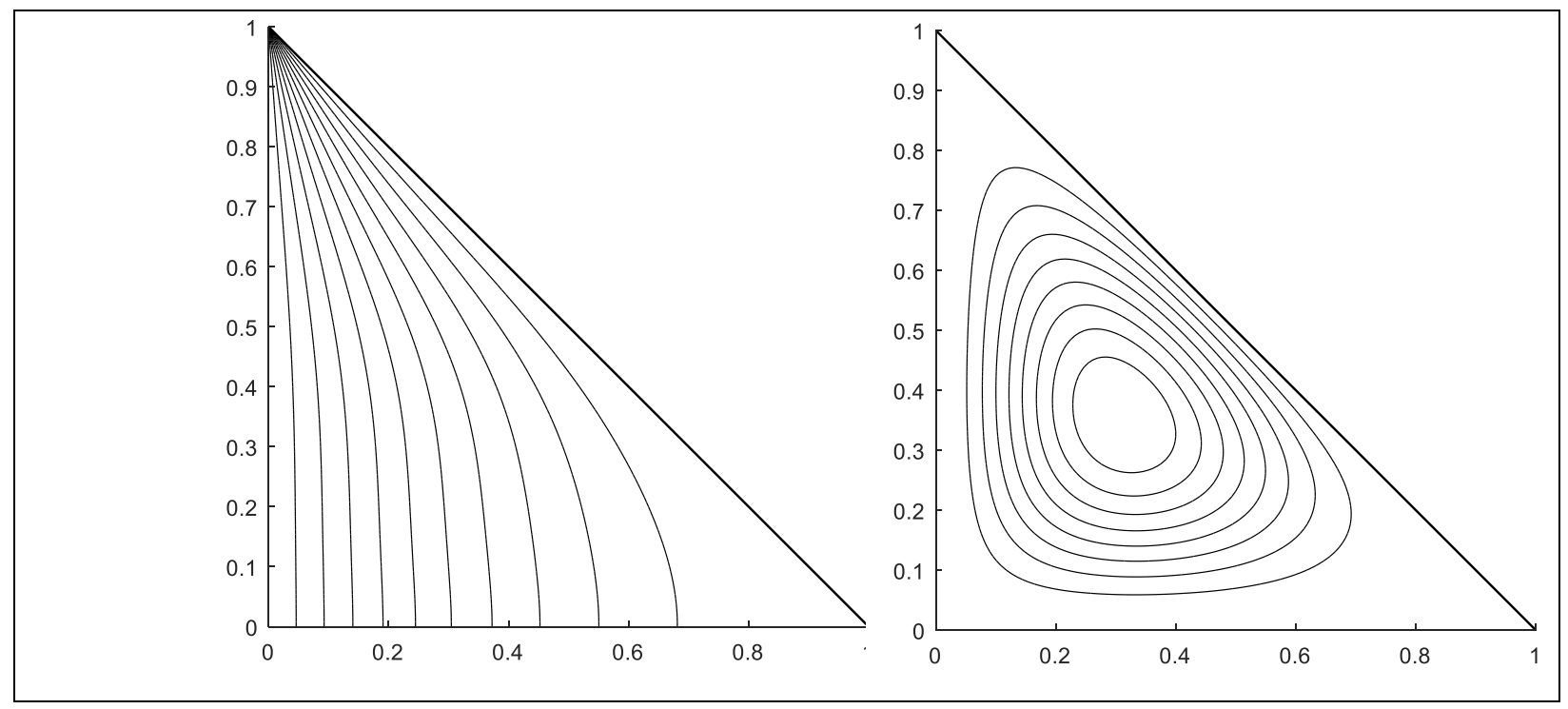

Fig. 2 Comparison of present work (Bottom - FDM) and FEM solution of Basak et al. [37] (Top GFEM) of contour plots for uniformly heated left vertical wall, $\theta(0, Y)=1$ and cooled right inclined wall, $\theta(X, Y)=0, \forall X+Y=1$ and insulated bottom wall with $R a=10^{3}$ and $\operatorname{Pr}=0.7$ (air).

$\mathrm{Da}=0.0001$
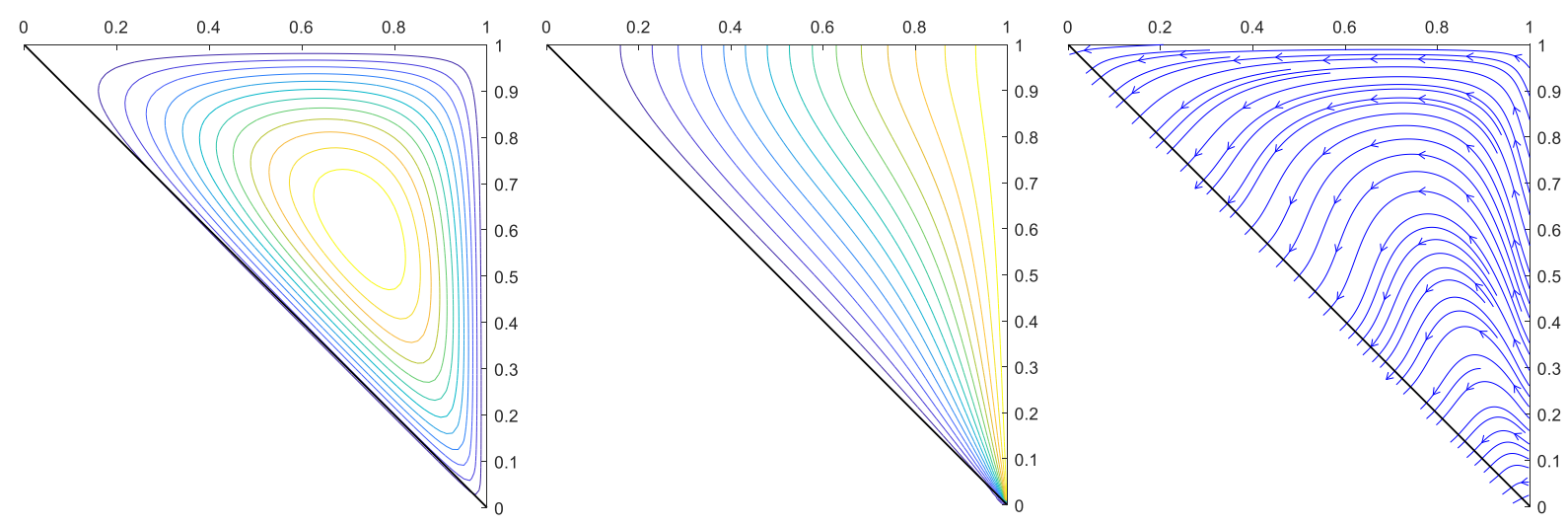

$\mathrm{Da}=0.001$ 

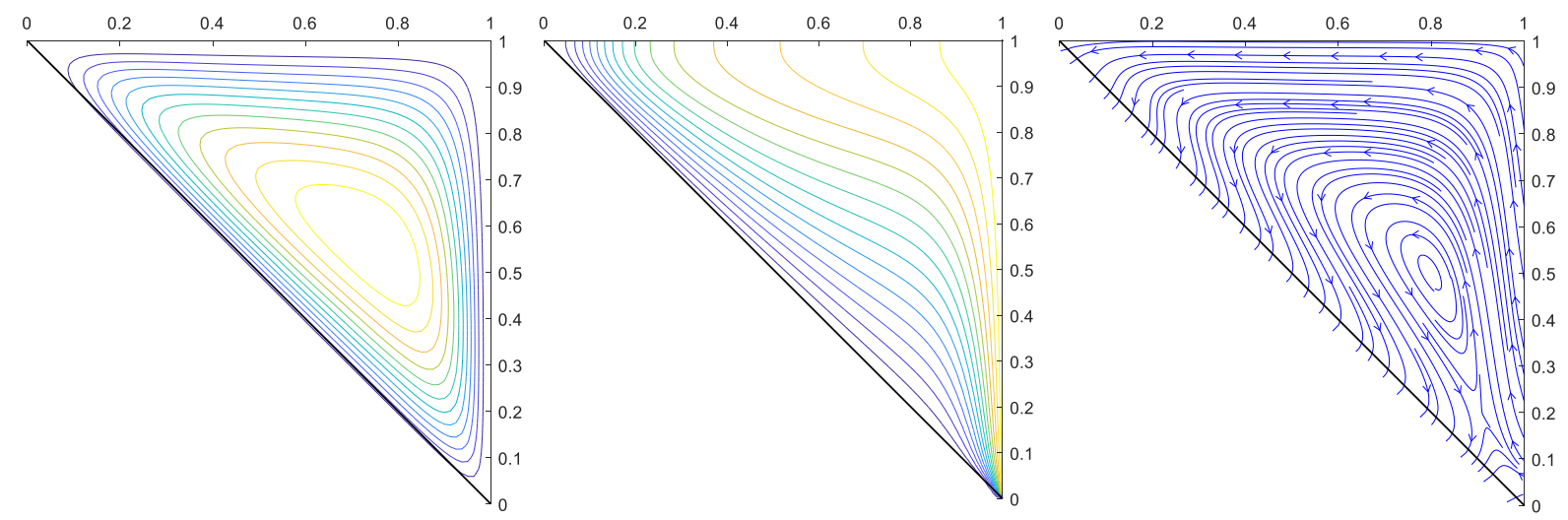

Fig 3. Streamlines, isotherms and energy flux vectors for $R a=100, R d=1, \varepsilon=1.2 \%$.

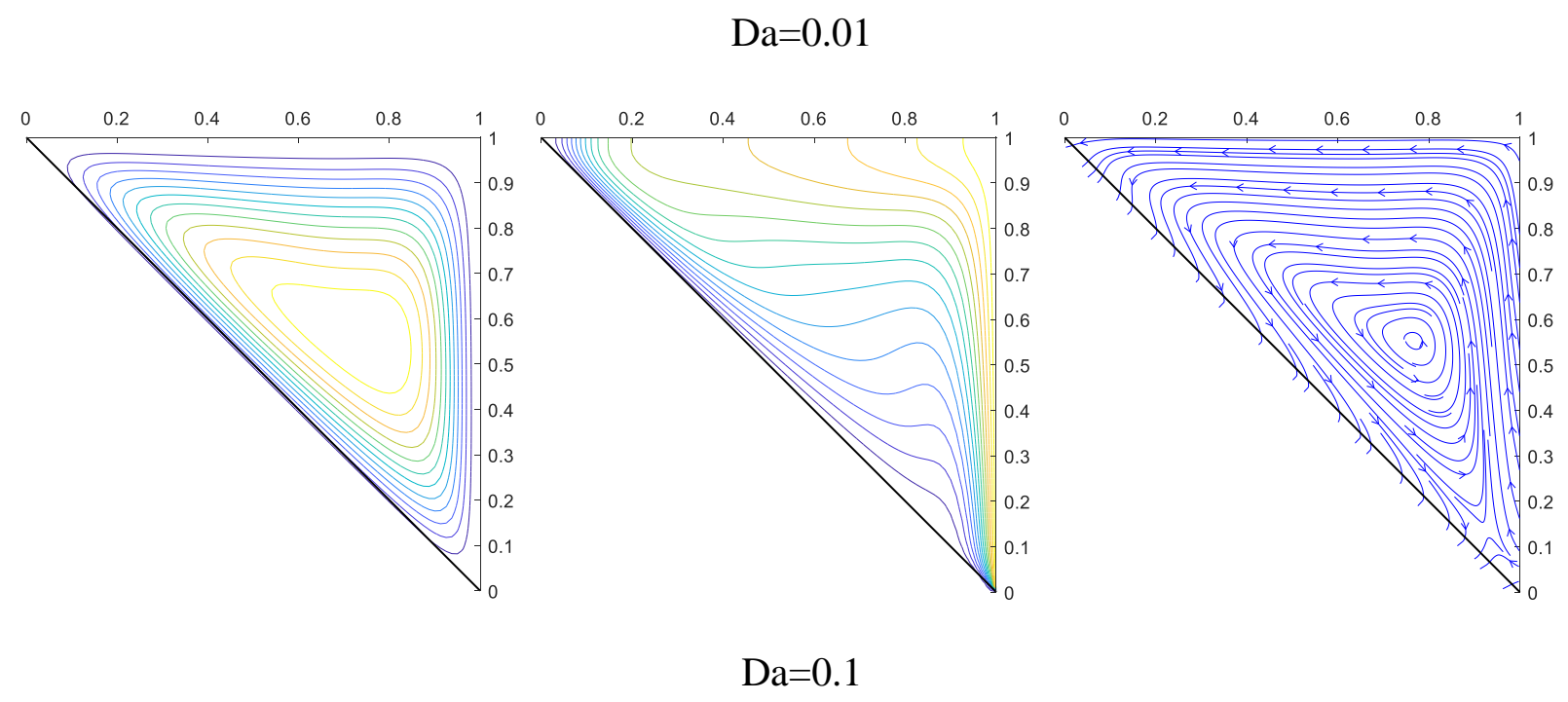



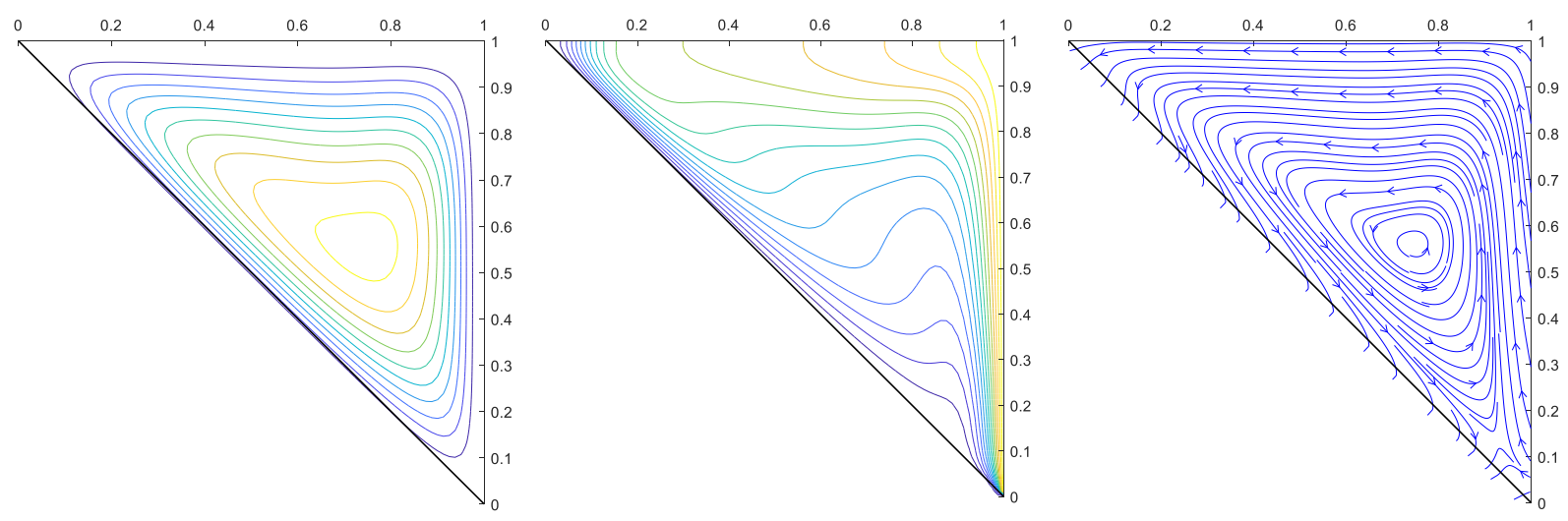

Fig 4. Streamlines, isotherms and energy flux vectors for $R a=100, R d=1, \varepsilon=1.2 \%$.

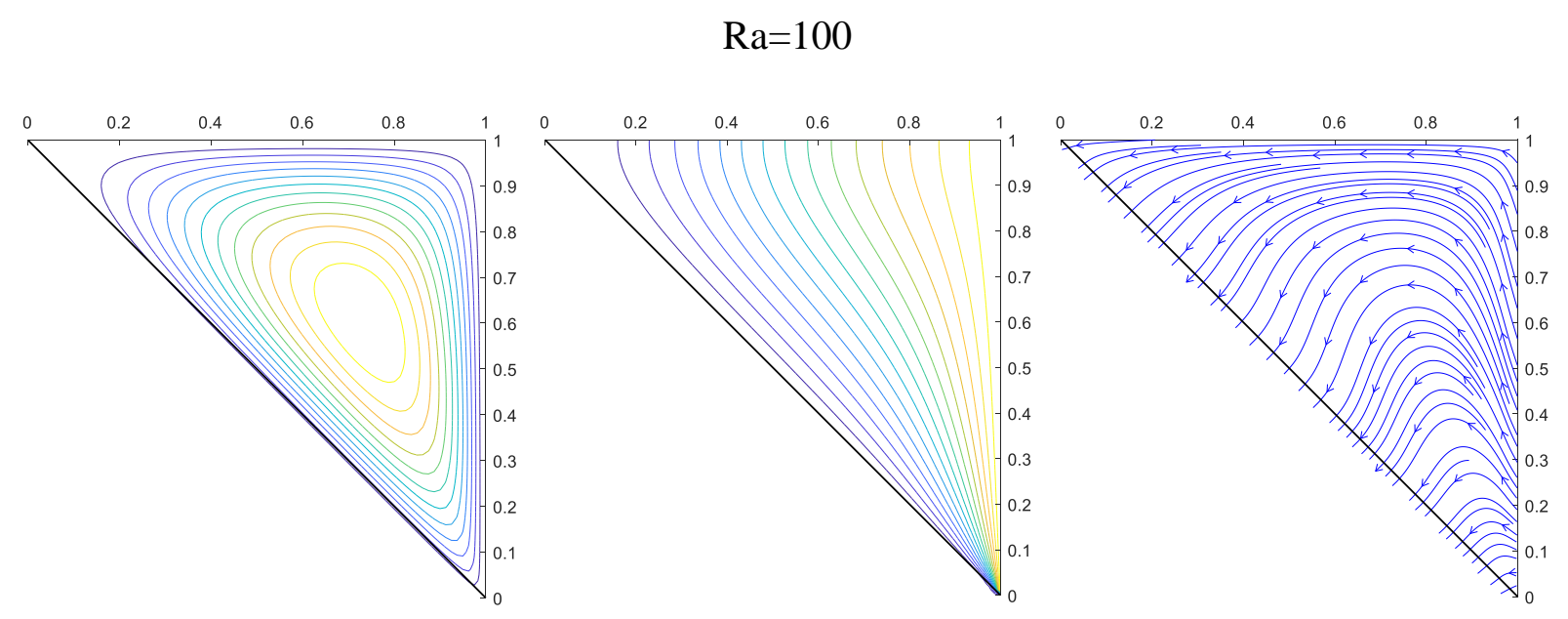

$\mathrm{Ra}=500$ 

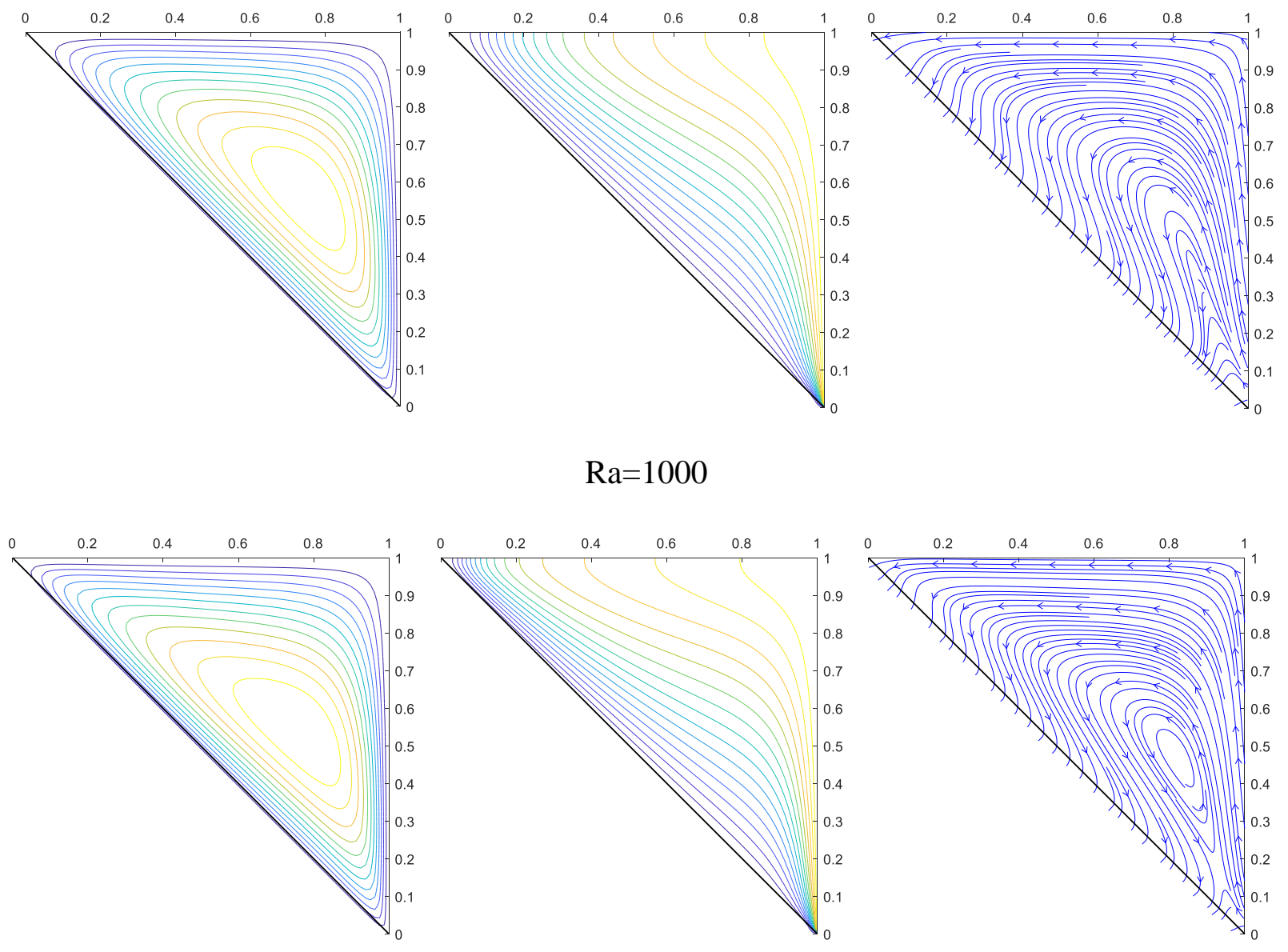

Fig 5. Streamlines, isotherms and energy flux vectors for $D a=0.0001, R d=1, \varepsilon=1.2 \%$. 


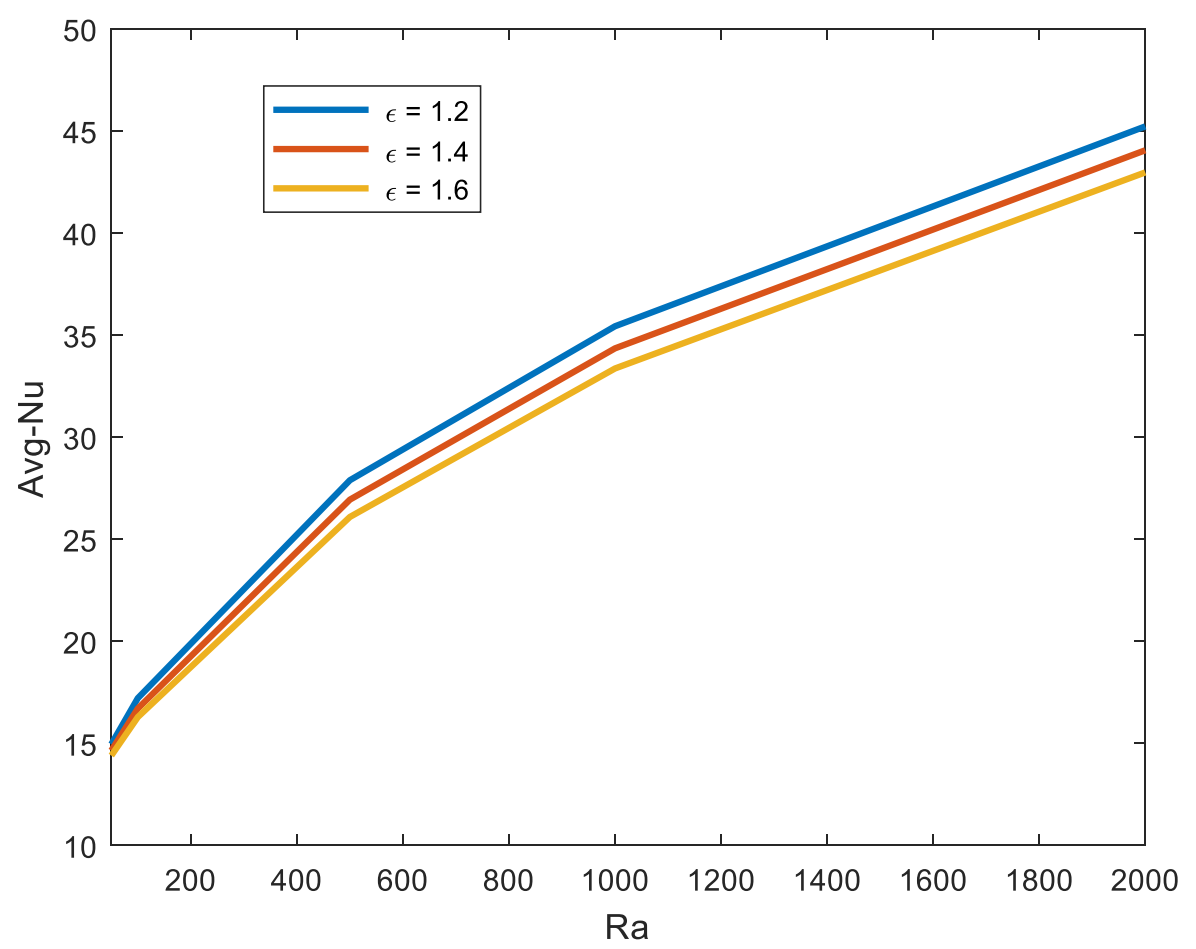

Fig 6. The average Nusselt number for $R a$ with $D a=0.0001, R d=1$.

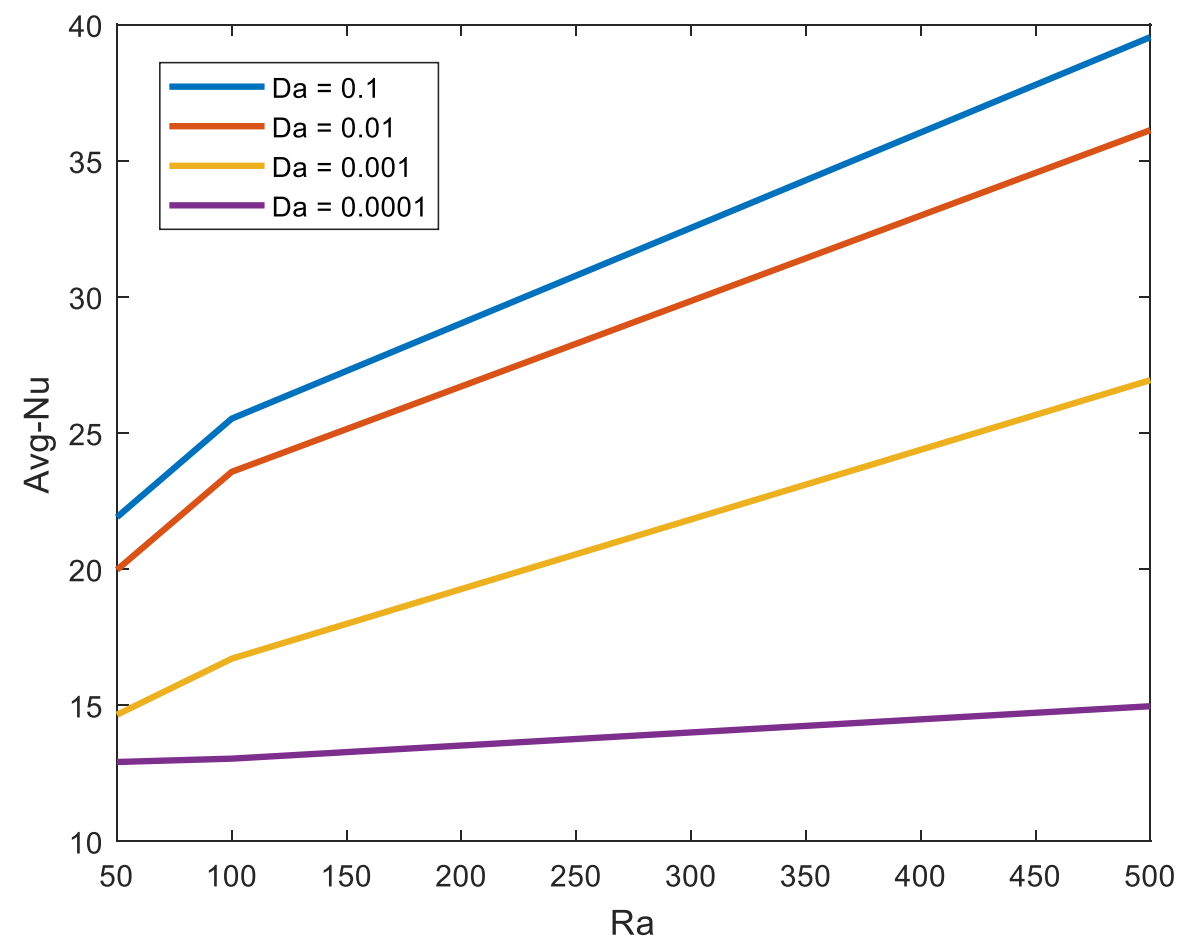

Fig 7. The average Nusselt number for $R a$ with $R d=1, \varepsilon=1.4 \%$. 


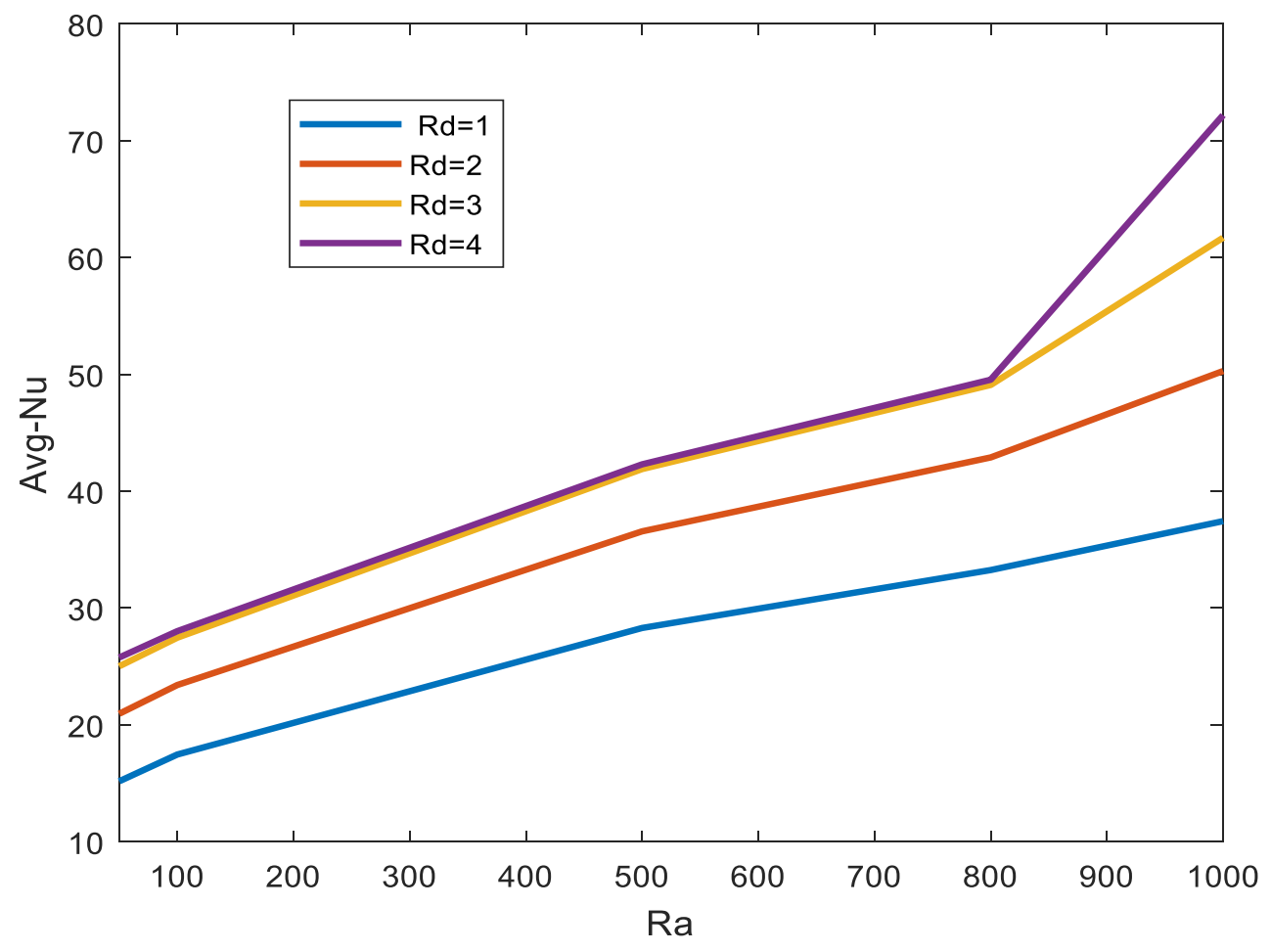

Fig 8. The average Nusselt number for Ra with $\varepsilon=1.2$, $D a=0.001$. 